\title{
La educación general transdisciplinaria y descolonial en una sociedad democrática ${ }^{1}$
}

\section{Transdisciplinary and decolonial general education in a democratic society}

Recibido: 3 de octubre de 2021 | Aprobado: 23 de noviembre de 2021

\section{Resumen}

Esta es una reflexión conceptual crítica de la educación general transdisciplinaria como vía promotora de la descolonialidad en una sociedad democrática. Se analiza holísticamente la importancia de la pluralidad e integración de saberes, experiencias, vivencias y valores de quienes participan en el proceso educativo, su consecuente resultado en praxis transformadoras y la potenciación de la ciudadanía en un contexto democrático emancipador. Filosóficamente, esta visión, transdisciplinaria y descolonial promueve una conciencia ética y de acción política compleja que requiere un examen crítico de la relación entre la enseñanza y el aprendizaje y todos sus componentes complejos. Es una propuesta de convergencia crítica hacia el advenimiento de una nueva forma de ser, pensar, hacer y sentir para quienes participan en este complejo proceso. Se discute tres áreas temáticas: (1) Educación general transdisciplinaria y democracia, (2) Pluriálogos, descolonialidad y vida democrática y (3) Descolonialidad, praxis democrática y educación general transdisciplinaria. Este trabajo tiene el fin de generar praxis transformadoras de la sociedad, donde el conocimiento sea democratizado, liberador y emancipador en el contexto de las condiciones en que se inserta en Latinoamérica y el Caribe. La conclusión de este trabajo lleva a considerar que la producción de nuevos conocimientos y la revaloración axiológica de la educación general desde la transdisiciplinariedad descolonial señalará posibles rutas para el ensayo de alternativas democráticas en atención de la complejidad social actual.

Palabras clave: Educación general, transdisciplinariedad, Complejidad, descolonialidad, vida democrática

1 Ponencia presentada en junio de 2021 en el XII Simposio Internacional de Estudios Generales (modalidad virtual). Pontificia Universidad Católica Madrey Maestra (PUCMM), República Dominicana, y Red Internacional de Estudios Generales (RIDEG).

* Catedrático retirado de la Universidad de Puerto Rico, recinto de Aguadilla. Posee un bachillerato en artes en Ciencias Políticas y una maestría en artes en Psicología Social Comunitaria de la Universidad de Puerto Rico y un doctorado en Psicología Ambiental de The Graduate School and University Center of the City University of New York. Para contactar al autor: juliov.montalvo@upr.edu

ISSN (en línea): 1814-4152 / Sitio web: http://cuaderno.pucmm.edu.do

Cómo CITAR: Montalvo Del Valle, J. (2022). La educación general transdisciplinaria y descolonial en una sociedad democrática. Cuaderno de Pedagogía Universitaria, 19 (37), 36-46. 


\section{Abstract}

This is a critical conceptual reflection of transdisciplinary general education as a promoter of decoloniality in a democratic society. The importance of plurality and integration of knowledge, experiences, significant life experiences and values of those who participate in the educational process, its consequent result in transformative praxis and the empowerment of citizenship in an emancipatory democratic context, is holistically analyzed. Philosophically, this transdisciplinary and decolonial vision promotes an ethical consciousness and complex political action that requires a critical examination of the relationship between teaching and learning and all its complex components. It is a proposal for critical convergence towards the advent of a new way of being, thinking, doing, and feeling for those who participate in this complex process. Three thematic areas are discussed: (1) Transdisciplinary general education and democracy, (2) Pluri-dialogues, decoloniality and democratic life and (3) Decoloniality, democratic praxis and transdisciplinary general education. This work has the purpose of generating transformative praxis in society, where knowledge is democratized, liberating, and emancipating in the context of the conditions in which it is inserted in Latin America and the Caribbean. The conclusion of this work leads to consider that the production of new knowledge, the axiological revaluation of general education from the decolonial transdisciplinarity will point out possible routes for the test of democratic alternatives in attention of the current social complexity.

Keywords: General Education, transdisciplinarity, complexity, decoloniality, democratic life.

\section{Introducción}

Este trabajo es una reflexión conceptual crítica de la educación general y su planteamiento transdisciplinar como vía promotora de la descolonialidad en una sociedad participativa y democrática. Los temas son abordados desde una perspectiva holística considerando la importancia de los pluriálogos (pluralidad de diálogos), la integración de saberes, experiencias, vivencias y valores de quienes participan del proceso, su consecuente resultado en praxis transformadoras y la potenciación de la ciudadanía en un contexto democrático emancipador. De acuerdo con Rincón et al. (2015):

La decolonialidad como parte constitutiva de la matriz analítica modernidad/colonialidad, establece una relación de diálogo conflictivo que surge en el mismo momento que comienza el despliegue e imposición planetaria de la retórica salvacionista moderna, su visión prepotente del mundo, así como el ejercicio exagerado del poder, constituyéndose esta última categoría en uno de los debates centrales de la red modernidad/colonialidad sobre la cual descansa buena parte de la reflexión que da como derivación el proyecto decolonial. De igual manera, han sido otras las discusiones que han mantenido ocupados a los llamados pensadores de la red modernidad/colonialidad, conceptos que explican el comportamiento, intenciones, prejuicios del poder imperial hasta aquellos que se presentan como una alternativa justa y comprometida éticamente con la paz y la dignidad de los pueblos del mundo, en otras palabras, conceptos como: colonialidad del poder, del ser, del saber, interculturalidad, transmodernidad son sin duda supuestos filosóficos, ontológicos y epistémicos clave dentro del proyecto decolonial (Rincón et al., 2015, pp. 81-82).

La educación general como vía descolonial debe asumirse de forma transdisciplinaria y aportar a un análisis develador de la colonialidad así como de sus formas de reproducirse en las actividades de los procesos de enseñanza y aprendizaje, en los programas de estudio (colonialidad curricular), en las prácticas docentes tradicionales y, más ampliamente, en las acciones prácticas cotidianas de la complejidad holística. Quienes participan en tal complejidad, deben ser agentes activos, conscientes de la integración de saberes, experiencias y propuestas transdisciplinarias. De esta manera, irían dirigidas hacia un proceso de transformación para deconstruir las relaciones estructurales de la colonialidad en la educación general y dar paso a alternativas basadas en las praxis democráticas descoloniales. 
Dentro de esta visión, transdisciplinaria y descolonial, hay un posicionamiento ético y de acción política que requiere una toma de conciencia crítica de la correspondencia entre la enseñanza y el aprendizaje, la docencia y el conjunto de todos sus componentes complejos. Esta es una propuesta de confluencias críticas crítica hacia de una nueva forma de ser, pensar, hacer y sentir para quienes participan de este complejo proceso en la Universidad y más allá de ella. Va encaminada a generar praxis transformadoras de la sociedad, donde el conocimiento sea democratizado, liberador y emancipador en el contexto de las condiciones históricas, sociales y culturales en que se inserta.

Es un compromiso fundamental de atender asuntos de vigencia, pertinencia y urgencia por parte de las sociedades mundiales actuales, que atañen a las personas, comunidades y otras configuraciones históricas, sociales, políticas y culturales. Son parte de la complejidad social la sustentabilidad de la vida, lo ambiental y ecológico, la salud humana y del planeta, la crisis cultural, la globalización, las guerras, el patriarcado, el tema del género y sus ramificaciones (feminicidios, la educación con perspectiva de género y otras) por un lado; las drogas, la corrupción y las policrisis, entre otros, que requieren un posicionamiento ético transdisciplinario y descolonial como elementos de la educación general universitaria, por el otro. También incluye la consideración de una conciencia ciudadana que fluya desde la pluralidad y se asuma en un discurso democrático y descolonial. Todo esto fundamentado en la praxis transformadora ante el poder acaparador de los Estados y gobiernos todopoderosos promotores de la inequidad, desigualdad económica y la injusticia social.

Ante la complejidad social, la educación general universitaria suele reflejar amplias y diversas tendencias históricas, sociales y culturales. Estos marcos de referencia señalan posibles rutas a seguir en la investigación y aplicación práctica de la educación general.

En ellos se entremezclan manifestaciones vitales junto a concepciones epistemológicas, metodológicas, ontológicas, teóricas y prácticas impartiéndole una dinamicidad única a los diversos quehaceres y prácticas docentes, en y fuera del salón de clases.
El tema de la descolonialidad adquiere un relieve particular al vincularlo al tema de la transdisciplinariedad en el contexto de la educación general en tanto posibilita la pluralidad de "diálogos", de pluriálogos, entre distintas áreas del conocimiento que producirá nuevas epistemes. Esta exposición a campos del saber inherentes a los problemas y retos de la educación general presta atención a la pertinencia y la diversidad de modos de organizar efectivamente las prácticas docentes universitarias en América Latina y el Caribe,

Tanto el pensamiento descolonial como el Paradigma de la Complejidad y la transdisciplinariedad desmontan transgresivamente el discurso de la modernidad que aún sigue presente en la educación general universitaria manteniendo nociones de universalismo eurocéntrico. Es menester hacer, pensar y sentir de maneras diversas, formas de transformación, emancipación y transgresión en todos los órdenes de la vida y la existencia desde el profundo autoexamen y asumir posicionamientos en los distintos planos de la complejidad de nuestra existencia y conciencia vital.

Por ello viene al caso el planteamiento de Morin sobre la transdisciplinariedad como resultado histórico de las imperfecciones crecientes en los modos dominantes de construir el conocimiento desde aproximadamente tres siglos. Esta es una manera de organización de los conocimientos que trascienden las disciplinas de una forma radical. La transdisciplina nos invita a procurar un conocimiento lo más completo posible, aquel que puede comprender el mundo desde todas las perspectivas y dialogar con la diversidad de los saberes humanos. Apunta a ese diálogo cuestionante que impera en toda mente que aspira la búsqueda compleja de un saber que transciende a una disciplina en particular con el fin de comprender el mundo desde la unidad del conocimiento, a sabiendas de que se trata de un conocimiento inacabado, relacional y complejo. (Morin, 2018).

Transdisciplina y complejidad están estrechamente unidas como formas de pensamiento relacional y como interpretaciones del conocimiento desde la perspectiva de la vida humana y el compromiso social. Nicolescu (1996) plantea: 
La imperiosa necesidad de proponer, vivir, aprender y enseñar un pensamiento complejo, que vuelva a tejer las disciplinas como posibilidad de humanidad en completud (sic); y que sólo de esta manera se vencería la eterna limitación y fragmentación del sujeto separado de sí mismo en la búsqueda del conocimiento. (Nicolescu, 1996, en Morin, 2018, p.3).

Siendo así la transdisciplina, el análisis de la complejidad y el pensamiento decolonial o descolonial forman una unidad compleja epistemológica, ontológica, metodológica y práctica. Esta unidad es parte fundamental de la educación general, dentro y fuera del ambiente universitario. Es requerida para la construcción de nuevos marcos analíticos y la promoción de praxis democráticas transformadoras de la sociedad.

Algunas instancias donde se puede ver concretamente la construcción de estas gestiones son el trabajo comunitario en el que grupos de ciudadanos y ciudadanas colaboran conjuntamente con grupos de estudiantes universitarios realizando actividades donde ambos aprenden de la interacción y el trabajo realizado. Otra instancia es el trabajo cultural que realizan grupos de vecinos en cooperación con grupos universitarios de donde salen proyectos de creatividad y aprendizaje popular para fomentar la búsqueda de alternativas a asuntos de interés vecinal. No menos importantes son los proyectos de autogestión comunal a través de comedores sociales urbanos y cooperativas de alimentos donde personas de la comunidad y estudiantes universitarios se integran para compartir conocimientos, experiencias organizativas y aprendizajes vivenciales que luego forman bases para seguir mejorando la experiencia.

\section{Educación general transdisciplinaria y democracia}

Toda educación general transdisciplinaria como vía descolonial y democrática es un proceso multidimensional complejo en tanto histórico, social, económico, político, cultural y psicológico que atraviesa y transcurre en todos los aspectos de las vidas de sus participantes. Como vía promotora de la descolonialidad en una sociedad participativa y democrática se manifiesta transversalmente en su complejidad. Es holística y considera la importancia de los pluriálogos (o pluralidad de diálogos), la integración de saberes, experiencias, vivencias y valores de docentes y aprendices y otros participantes. Desde un punto de vista práctico, deberá resultar en praxis transformadoras y potenciadoras de dinámicas democráticas emancipadoras.

La educación general como instrumento descolonial transdisciplinario debe aportar al análisis develador de la colonialidad, a sus formas de reproducirse en los procesos de enseñanza y aprendizaje, en los currículos y en las prácticas cotidianas de la complejidad holística (Castro-Gómez, 2007). Quienes participan en tal proceso complejo deben ser agentes activos y conscientes en la integración de saberes, experiencias, propuestas inclusivas y transdisciplinarias que abarquen transformaciones para deconstruir las relaciones estructurales de la colonialidad en la educación general y dar paso a dinámicas democráticas y participativas.

De esta visión, emana una conciencia ética y de acción política que produce un examen crítico de la relación entre la enseñanza y el aprendizaje y todos sus componentes complejos. La educación general transdisciplinaria descolonial es una propuesta de convergencia crítica hacia el advenimiento de una nueva forma de ser, pensar y hacer de sus participantes. Como propuesta genera praxis transformadoras de la sociedad, donde el conocimiento es democratizado, liberador y emancipador en el contexto de las condiciones particulares desde donde se inserta considerando la vida humana y el compromiso social.

La comprensión de lo complejo en distintas dimensiones, niveles de realidad y sus relaciones holísticas en el contexto total de la educación general es de vital importancia. Es la propia complejidad social la que impulsa a encontrar soluciones a problemas complejos desde la transdisciplinariedad y la descolonialidad. La transdisciplinariedad es una respuesta a los cambios rápidos, múltiples que caracterizan la complejidad de las sociedades de los siglos $X X$ y XXI. La educación general transdisciplinaria y descolonial puede y debe ser un instrumento para gestar nuevas formas de desenvolverse en la vida de las sociedades democráticas. 
Promover valores de una vida democrática a través de estudios y prácticas pensadas con ese fin es darles poder a sus participantes en el proceso educativo para devenir en aprendizajes transdisciplinarios que integren saberes académicos formales, sabidurías populares y discursos subalternos en nuevas epistemes. Ello conlleva praxis consensuadas para convivir respetando la diversidad y atendiendo las dimensiones inmediatas y mediatas de la complejidad social.

La puesta en práctica de la integración de nuevos conocimientos, la utilización de métodos de investigación transdisciplinaria y sus resultados aplicados a nivel cotidiano, supone transformaciones de las condiciones sociales, históricas, políticas y culturales de la colonialidad. Como mínimo requiere llevar a cabo una educación holística de personas autónomas, con conciencia analítica y crítica que ejerzan prácticas de convivencia y consensos colectivos en la praxis democrática. Esta vida y acción democrática descolonial se afianza en la educación transdisciplinaria que empodera a la ciudadanía y le potencia como sujetos cambiantes, activos, emancipados y humanos con nuevas cualidades analíticas, emocionales y prácticas.

Una educación general transdisciplinaria y descolonial supone entendimiento holístico de las relaciones entre las partes que la componen y el todo que lo conforma en "lo complejo, complexus, o lo que está tejido en conjunto” (Morin, 2013). Implica comprender procesos históricos, sociales, económicos, políticos, culturales colectivos, así como los psicológicos individuales que dan forma y transcurren por todos los aspectos de las vidas de quienes participan en ella, sus interrelaciones e interconexiones. También debemos verla como un vehículo para atender y enfrentar los retos que la complejidad social plantea por medio del acceso al conocimiento producido por quienes son parte del proceso educativo en su contexto y así como en diversos escenarios concretos, que permitirán entender nuevas formas de acción, pensamiento y de inserción en la vida digna, plena, descolonizada y democrática.

La educación general transdisciplinaria y descolonial universitaria debe ser un instrumento para que el estudiantado se empodere para enfrentar retos que la complejidad social plantea en su inmediatez y en su mediatez. Mediante el acceso al conocimiento producido por quienes son parte del proceso educativo en su contexto y en diversos escenarios concretos se logrará entender nuevas formas de acción, pensamiento y de inserción en la vida, digna y plenamente. Nicolescu (1996) plantea que para la producción del conocimiento transdisciplinario se necesita de una mente abierta durante el proceso de aprendizaje. Esta apertura involucra una relación con la sociedad civil, con el tiempo del ciberespacio y una consideración de la universalidad con el fin de redefinir los valores que rigen la propia existencia. Es de notar la gran pertinencia para la práctica docente en la educación general transdisciplinaria que tienen estos señalamientos. Nuevos y múltiples procesos de aprender dirigidos hacia la sociedad civil, hacia un lugar no físico donde se da en el tiempo cibernético el propósito de la universidad y de la educación general y hacia una valoración sobre la propia existencia y la vida.

Pluriálogos, descolonialidad y vida democrática

El tema de la colonialidad adquiere relieve particular al relacionarlo con el de la transdisciplinariedad en el contexto de la educación general universitaria en la medida que posibilita pluriálogos entre distintas áreas del conocimiento y produce nuevas epistemes. Esto contribuye a la exposición a diversos campos del saber y presta atención a la pertinencia y maneras de organizar los componentes de las prácticas docentes universitarias en América Latina y el Caribe. Ello ocurre en la medida en que hacer, pensar y sentir diversas formas de transformación, emancipación y transgresión se van dando en el proceso democrático de educación general.

Viene al propósito examinar lo que es colonialidad por su pertinencia al desarrollo de este análisis. Este concepto se deriva de colonia. Una colonia es un territorio sujeto a la administración y gobierno de otro país, por lo general, una potencia remota llamada 'metrópoli', que ha invadido y sometido a sus habitantes por la fuerza ${ }^{1}$, por lo cual, generalmente, carecen de autonomía y autodeterminación. Un 
claro ejemplo caribeño de lo que es colonia es el caso de Puerto Rico, una colonia bajo el imperio español desde 19 de noviembre de 1493, hasta el 25 de julio de 1898 en que se produce la invasión militar de los Estados Unidos, hasta el presente donde se manifiestan características transmodernas complejas de colonialidad.

El concepto colonia hace referencia histórica a la apropiación llevada a cabo por España de los territorios que conocemos como América. Apropiación ejecutada al final de la conquista como proceso bélico y genocida que comenzó hacia el siglo XVI, hasta el XIX, a lo largo del cual las nuevas naciones americanas fueron logrando su independencia política de la España (Historiografía, 2017)2. En párrafos posteriores se abunda en la participación histórica de otros imperios coloniales europeos en la llamada conquista de "América".

La categoría colonialidad o colonialidad del poder designa al patrón estructural de poder específico de la modernidad, originado a partir de la conquista de América y la subsecuente hegemonía planetaria europea (Quintero en CECIES, 2010-2019). Fueron las ideas de Anibal Quijano, sociólogo peruano, las que redefinieron la categoría de "colonialidad", la cual surge a partir del marco de interpretación de la modernidad bajo el prisma histórico y cultural de Latinoamérica. Quijano (1992) la cataloga como "el nodo epistémico" para estructurar el poder hegemónico sobre Latinoamérica. Los orígenes de esta categoría remiten directamente a las investigaciones y propuestas teóricas de Quijano, progenitor de la noción en América Latina (Quijano, 2000a y 2000b; Quijano, 2001; Quijano, 1991, 1992; Quijano y Wallerstein, 1992)

El binomio colonialidad del poder es una noción sostenida por Quijano (1991, 1992, 1993, 2000ª 2000b) cuyo fin es ofrecernos los esquemas de dominación global propios del sistema-mundo moderno/capitalista originados con la conquista de América en el siglo XVI y denominado el colonialismo europeo. Inicialmente, esta hegemonía estuvo bajo el puño del dominio español y, a los pocos años, se unió el imperio portugués. Más adelante, en el siglo XVIII el colonialismo pasó a la hegemonía francesa y holandesa, y también a la par, se hace notorio el imperialismo inglés que se perpetúa con gran domino en el siglo XIX. Por último, América Latina ha sufrido los embates del dominio imperialista estadounidense desde principios del siglo XX hasta hoy en día. Así las cosas, la colonialidad del poder incluye una larga lista de transformaciones y transmutaciones de las dimensiones subjetivas (clasificaciones sociales) y materiales (formas de control del trabajo) de este patrón. (Quintero, 2010).

Rojas Tudela (2017), a partir de las ideas de Quijano, explica que la colonialidad amerita un entramado de relaciones sociales intersubjetivas en el que el dominador reconoce y necesita del dominado con el fin de ejercer su dominio e instaurar su sistema de explotación, así como para establecer la clasificación social jerárquica que consiste en la articulación de todas las formas conocidas de expropiación del trabajo en una única estructura hegemonizada por el capitalismo. Así, la colonialidad representa uno de los elementos constitutivos del patrón global de poder capitalista.

Resultan interesantes los aportes de Quintero (2010) sobre el fenómeno político-social independentista que se inicia en el siglo XIX en la mayoría de las colonias de América y su efecto en la colonialidad. Pudiera pensarse que con las independencias se lograría eliminar la subyugación, ya que su principal objetivo fue la emancipación. Sin embargo, este fue un proceso de descolonización, pero no de descolonialidad. De acuerdo con Quintero (2010), "los nuevos estados-nacionales latinoamericanos logran independizarse de las potencias hegemónicas, pero la colonialidad y sus efectos fundamentales siguen operando a lo interno de los distintos países". A pesar de toda la sangre derramada, en América Latina la colonialidad del poder sigue siendo el elemento central de la estructuración de la sociedad.

La colonialidad del poder, según Quijano, es, sin dudas, uno de los ejes que estructura el capitalismo colonial moderno. Podríamos pensar que esta noción mayormente afecta al sistema laboraleconómico, pero no, su mayor impronta que ha trascendido la relación colonial original consiste en la clasificación social jerárquica de la población latinoamericana según criterios raciales, lo cual ha

2 (http://www.la-razon.com) 
afectado a todas las manifestaciones del poder mundial poscolonial.

En la última década se ha generado una honda expansión de la noción de colonialidad y de sus usos. Aún más allá de América Latina podemos encontrar el concepto en distintos estudios. Según Escobar (2005), "el uso más extendido y significativo de la propuesta de la colonialidad está ligado a lo que se ha conocido como el "proyecto modernidad/colonialidad/decolonialidad' que reúne a un conjunto heterogéneo de intelectualesactivistas latinoamericanos en torno al debate sobre estas problemáticas". El autor considera que en las elaboraciones de Quijano el concepto colonialidad y colonialidad del poder son permutables, lo que no sucede con en las producciones posteriores de otros autores quienes han propuesto distintos usos y flexiones del término colonialidad con el fin de profundizar y enfocar diferentes ámbitos o problemáticas. Entre las flexiones más extendidas de la noción se encuentran: colonialidad del saber, colonialidad del ser y colonialidad de la naturaleza.

Debe notarse que

el concepto de colonialidad del poder difiere de la noción de "colonialismo". Colonialismo designa una relación política y económica, en la cual la soberanía de un pueblo reside en el poder de otro pueblo o nación que explota la naturaleza y productos del trabajo de los colonizados. En contraposición a esto, la colonialidad se refiere a un patrón de poder que emergió como resultado del colonialismo moderno, pero que en lugar de estar limitado a una relación de poder entre dos pueblos o naciones, más bien a la forma como el trabajo, el conocimiento, la autoridad y las relaciones intersubjetivas se articulan entre sí a través del mercado capitalista mundial y de la diferencia colonial. Así, pues, aunque el colonialismo precede temporalmente a la colonialidad, la colonialidad, en tanto matriz de poder, sobrevive al colonialismo (Quintero, 2010, p. 9, en nota al calce).

Todo lo anterior expuesto nos permite concluir que la idea de colonialidad no queda limitada al concepto de colonialismo, es decir, el dominio de la metrópoli no acaba en la independencia de la colonia. De esta manera, la noción de colonialidad apunta a las diferentes maneras y formas en que se mantiene y se prorroga la colonia después de la colonia, mejor dicho, la dominación después de que se dice que esta cesó o se fue.

Siguiendo la misma idea, cabe señalar que la categoría colonialidad no designa simplemente a una "herencia" colonial, sino que se trata de un modelo estructural de dominación, explotación y conflicto originado con el colonialismo global europeo. De esta manera, la colonialidad, como patrón de poder, sobrevive al colonialismo y ha sido reconfigurado constantemente durante los cinco largos siglos desde el origen de la conquista. De esta manera, la emancipación latinoamericana del siglo XIX desmanteló al colonialismo, pero no a la colonialidad, cuyo patrón de poder consiste en el sistema ordenador y acumulativo de las relaciones sociales evidente en la trama histórica de América Latina.

Desde el punto de vista descolonial, Dussel (2013) también presenta elaboraciones de importantes consideraciones con la teoría del giro descolonizador en la que critica la noción de la postmodernidad de pensadores europeos por eurocéntricas y la reemplaza por la transmodernidad, ir más allá de la modernidad. Él resume en cinco puntos los elementos centrales de esta teoría: 1. el giro descolonizador como un giro epistemológico; 2. un nuevo método filosófico: lo analéctico, una anadialéctica; 3 . la crítica a la modernidad y al eurocentrismo; 4. una nueva visión de la historia; 5. y una nueva política.

La descolonialidad como herramienta analítica transdisciplinaria produce transformaciones sociales y prácticas democráticas participativas que deben impulsarse desde la educación general transdisciplinaria en el contexto de los países latinoamericanos y caribeños y otras partes del sistema-mundo.

Descolonialidad, praxis democrática y educación general transdisciplinaria.

La transdisciplinariedad debe ser descolonial como sistema de pensamiento complejo. Una educación general universitaria transdisciplinaria ha de ser descolonial para ser democrática y promotora de la transformación social y de prácticas emancipatorias. 
Dussel (2013) concibe la democracia como "la voluntad de vida de un pueblo". Se trata de la capacidad del pueblo para participar en todas las decisiones, incluyendo las decisiones legales y políticas. Esta concepción que ofrece Dussel (2013) redefine el poder positivamente, ya que se trata de un concepto simétrico que concibe la vida en la factibilidad. Según el autor, significa querer vivir en libertad para tener cultura propia lo que incluye el derecho a tener formas de creencias, y no a una cosmovisión impuesta. Vivir también es querer comer, querer tener agua y poder elegir el sistema de gobernanza.

Además, es coincidir consensualmente en todas las decisiones. La hegemonía es el único proyecto que la política puede formular porque un proyecto unánime es imposible. La sede del poder es de la comunidad política y en concreto, históricamente, el pueblo, que no es toda la comunidad política sino los que se hacen actores del cambio de la historia (está también el anti-pueblo) (Dussel, 2013, p. 10).

Desde este punto de vista y aplicado a la educación general descolonial, ésta adquiere un carácter político amplio donde quienes participan del proceso tienen la voluntad de fomentar pluriálogos para realizar acciones consensuadas y modificar los enfoques y quehaceres tradicionales establecidos. La educación general transdisciplinaria incorporará las ideas colectivas que sirvan para promover y producir nuevos epistemes, praxis y metodologías vinculadas a la descolonialidad. De esta complejidad saldría lo que contribuiría a la voluntad de vida de un pueblo de la que habla Dussel.

El pensamiento descolonial es un pensamiento complejo y transdisciplinario que recoge el desarrollo del concepto en todas las facetas del conocimiento, la acción política que valida las experiencias y vivencias sociales e históricas en distintas partes del mundo. Es un punto de partida que revalora las nuevas perspectivas epistemológicas, ontológicas, axiológicas, éticas y prácticas del sistema mundo transmoderno y le da un nuevo sentido a la complejidad de la vida social y democrática.

Las prácticas docentes dirigidas a la descolonialidad deben darse en el contexto de los saberes populares, los saberes ancestrales, las visiones feministas, las perspectivas ecológico-ambientales y la diversidad de puntos de vista, experiencias y vivencias de quienes participan en el proceso de la educación general latinoamericana, caribeña y mundial como elementos constitutivos de un nuevo paradigma descolonial para la educación general universitaria. Sin embargo, es fundamental superar el eurocentrismo y la occidentalización como modelos fundacionales de la educación general. El pensamiento descolonial requiere de una educación general contextualizada y situada geopolítica, histórica, social y culturalmente, en la realidad latinoamericana y caribeña. La transdisciplinariedad admite y acepta la coexistencia de diversas epistemes, posibilita y promueve pluriálogos de saberes y sentires dentro y fuera de la Universidad. También es preciso revisar críticamente los planes de estudios o currículos que permitan posturas éticas y acciones prácticas correspondientes a esta forma de docencia descolonial y transdisciplinaria. Requiere que los programas curriculares tomen en seria consideración e importancia los contenidos curriculares formales, las vivencias y los trasfondos experienciales que traen los estudiantes en su bagaje vital al momento de entrar a la universidad. Ello representaría el abandono de la colonialidad curricular.

Desde el punto de vista transdisciplinario, es en el plan de estudios donde se manifiesta la importancia de las disciplinas, su insuficiencia y limitaciones para la producción de conocimientos desde la educación general universitaria que recalca la importancia del uso de la razón sobre otras características de los seres humanos. Se ignora la subjetividad, la afectividad, el amor y el arrepentimiento en sus propias vidas, poseedoras de un componente irracional importante. Esto no es así en la cosmovisión andina ni en la africana, donde la experiencia juega un papel fundamental en tanto productora de conocimientos (Vélez Cardona, 2014).

El respeto a la diversidad de cosmovisiones y al papel de nuestra propia subjetividad en el proceso de sentir/pensar es básico en una docencia transdisciplinaria que transgrede los cánones y acepte formas de saber y conocer que habían sido negadas por la academia occidental, colonial y moderna. Esto no significa obliterar la ciencia o los saberes occidentales modernos, sino de llevar a cabo una integración epistémica. "Un pensamiento 
integrativo en el que la ciencia occidental pueda "enlazarse" con otras formas de producción de conocimientos”. (Castro Gómez, 2007, p. 87)

Se trata de dar espacios igualitarios y significativos a los diferentes conocimientos y de ampliar las discusiones epistemológicas, así como maximizar la contribución de cada uno de ellos en la construcción de una sociedad más democrática, justa y más armónica en su relación con la naturaleza y con el planeta. Es permitir una discusión pragmática entre criterios alternativos de validez que no descalifique de una vez todo lo que no cabe en el canon epistemológico de la ciencia moderna (Boaventura de Sousa Santo, 2007, en Vélez Cardona, 2014).

También es importante contextualizar e identificar el lugar desde donde se formula el conocimiento al analizar la aportación de diversos saberes producidos a través de la historia ya que son saberes situados. El mal llamado "universalismo" (Maldonado Torres, 2007; Mignolo, 2000; Dussel, 2013), es una noción generada en Europa Occidental y promovida como exclusiva, válida y legítima en tanto fuente de saber, porque es la única que sigue los patrones de la racionalidad "científica", impulsada fundamentalmente a partir del Siglo XVIII por hombres blancos de apenas cinco países del mundo (Alemania, Francia, Italia, Inglaterra y Estados Unidos) (Vélez Cardona, 2014).

Al considerar la práctica docente hay que tomar en cuenta el elemento curricular y admitir que los currículos son manifiestos donde se plasman intencionalidades educativas sobre el perfil de ser humano que se pretende desarrollar. Explicitar las intencionalidades y asumir posturas éticas en relación con las prácticas docentes y los procesos de producción de conocimientos es un compromiso ineludible de la educación general transdisciplinaria y descolonial.

La idea de la práctica docente transdisciplinaria implica una amplia noción política y denota una estrecha relación entre saber y poder. La frase "el conocimiento es poder" (knowledge is power) mencionada en círculos académicos en décadas anteriores subraya el carácter de esta relación. Por lo tanto, posicionarse éticamente desde la producción de un conocimiento situado es acceder al poder para lograr transformaciones en el mundo real, de afirmación de la vida en la factibilidad (Dussel,2013).
Una educación general situada en el Caribe remite al pensamiento de varios pensadores, entre los que se destacan los martinicenses Aimé Césaire y Franz Fanon. Vélez Cardona (2006), defensor perenne de la renovación y el fortalecimiento de los Estudios Generales en el Caribe y Latinoamérica, cuestiona al eurocentrismo y se apoya en las ideas de Césaire, quien propone "que será del África negra, la madre de nuestra cultura y nuestra civilización antillana, de la que provendrá la regeneración de las Antillas; no de Europa, que solo puede perfeccionar nuestra alienación, sino de África, que es la única que puede revitalizar las Antillas". (Césaire, 2006 en Vélez Cardona, 2014).

El Caribe resulta ser un buen punto de partida para iniciar una nueva visión de los Estudios Generales. El pensamiento caribeño es aún más complejo que el resto de los países de América Latina dado que se origina de cosmovisiones diversas: europea, africana, autóctona de los pueblos que habitaban el Caribe, asiática y latinoamericana. La construcción de nuestra concepción del mundo partirá de esa realidad compleja. Según Vélez Cardona (2014), esta cosmovisión será:

no de manera provincial, sino todo lo contrario; sin exclusiones, censuras, represiones, ni jerarquías arrogantes. Lo haremos de manera participativa e incluyente, reconociendo las aportaciones de pensadores caribeños como Fanon, Césaire, Eduoart Glissent (Martinica), Michel-RolphTrouillot (Haiti), Paget Henry (Antigua), Lewis Gordon (Jamaica), Ochie Curiel (República Dominicana), MaldonadoTorres (Puerto Rico), Pedro Sotolongo (Cuba), entre muchos y muchas otras, incluyendo a las presentes. El desarrollo de unos Estudios Generales Latinoamericanos y Caribeños es un proyecto que requiere del concurso de múltiples voluntades.

\section{Conclusión}

Una educación general transdisciplinaria es necesaria como práctica de docencia democrática que trasciende la Universidad y se entrelaza con la vida activa de los ciudadanos y ciudadanas para producir transformaciones desde el compromiso social. La educación general transdisciplinaria descolonial es una herramienta para posibilitar la producción de conocimientos 
nuevos y metodologías para potenciar las praxis democráticas. La transdisciplinariedad es por definición democrática y el pensamiento descolonial igualmente promueve visiones democráticas del poder, del saber, del ser y de concepciones contextualizadas histórica, social, cultural y existencialmente. Ambas requieren ser agentes activos y conscientes de la integración de saberes, experiencias, vivencias y propuestas inclusivas que conduzcan a procesos de transformación política para deconstruir las relaciones estructurales de la colonialidad en la educación general y dar paso a alternativas dinámicas donde el conocimiento sea democratizador, liberador y emancipador en el contexto de las condiciones en que se inserta.

La puesta en práctica de la integración de nuevos conocimientos, la utilización de métodos de investigación transdisciplinaria y sus resultados aplicados a nivel cotidiano supone transformaciones de las condiciones sociales descoloniales a los que la educación general puede aportar significativamente dentro y fuera de la Universidad. La revisión crítica de los currículos disciplinarios y su transformación en transdisciplinarios y descoloniales ocurrirá mediante la integración de nuevas epistemes a través de pluriálogos y acciones democráticas producidas por los actores en el proceso. El empleo de metodologías participativas y un posicionamiento ético claro con respecto a los resultados que se desean obtener de esta práctica llevará a construir alternativas descoloniales, consensuadas y situadas en el contexto en que surgen.

Fuera del contexto de la educación general universitaria, es necesario fomentar el desarrollo de personas autónomas, con conciencia analítica y crítica que ejerzan prácticas de convivencia y consensos colectivos en la vida y la praxis democrática. Esta vida y acción democrática se afianza en la educación transdisciplinaria que empodera en las personas acciones consensuadas colectivas y les potencia como sujetos cambiantes, activos, emancipados y humanos totales con nuevas cualidades analíticas y prácticas descoloniales.

La transdisciplinariedad, la complejidad y el pensamiento descolonial son inseparables como elaboraciones conceptuales y como formas de acción humana que persiguen una valoración crítica de la vida y del humano enfrentado a las realidades que plantean las sociedades del siglo $X X I$. La reflexión ontológica, epistemológica y metodológica entran en juego para descubrir nuevas axiologías éticas y estéticas para integrar en el entendimiento y nuestra inteligencia emocional que dentro de la diversidad todos formamos un Todo complejo. Es en las relaciones e interconexiones de la multidimensionalidad y la aspiración a vivir y convivir pensando y actuando para el beneficio de los humanos, las cosas vivas, la ecología, el ambiente y la conciencia planetaria que se produce tal conjunción. Este análisis considera el pensamiento descolonial como forma de conocimiento transdisciplinario y medularmente democrático y que aporta a la comprensión de la complejidad en las distintas manifestaciones del poder, lo político, los elementos históricos, sociales y culturales como parte del contexto en que se da la educación general transdisciplinaria.

La educación general queda inmersa en esta forma de ver la complejidad de la realidad social y potencia el empoderamiento de los actores participantes en el proceso educativo transdisciplinario y descolonial. La dinámica de este proceder sitúa a la educación en el centro de acciones transformadoras transmitiendo y fomentando el desarrollo de cualidades de los sujetos en la vida democrática. Al fin de cuenta, la agenda auto impuesta es la de atender las complejidades de las sociedades contemporáneas y las polarizaciones geopolíticas que amenazan con destruir la vida en la colonialidad del poder según la conocemos en el capitalismo salvaje neoliberal, el cambio climático, la crisis de comunicación, es decir, las policrisis de nuestro tiempo.

La producción de nuevos conocimientos, la revaloración axiológica de la educación general desde la transdisciplinariedad descolonial señalarán posibles rutas para el ensayo de alternativas democráticas en atención de la complejidad social actual. Una educación general transdisciplinaria y descolonial situada en Latinoamérica y el Caribe lleva a tener en seria consideración y aprecio a las significativas aportaciones para los seres humanos que podemos ser y para ocupar un lugar digno con respeto a la vida, a las cosas vivas y a nuestro planeta. 


\section{Referencias bibliográficas}

Castro-Gómez, S. (2010). Transdisciplinariedad, latinoamericanismo y coIonialidad. Coloquio con el profesor Santiago-Castro-Gómez. Historia y MEMORIA, 181-192. https:/revistas.uptc.co/ index.php/historia memoria/article/view/782

Castro-Gómez, S. (2007). Descolonizar la universidad: La hybris del punto cero y el diálogo de saberes. En Castro-Gómez, S. y Gosfroguel, R. (Eds.) El giro decolonial. Reflexiones para una diversidad epistémica más allá del capitalismoglobal, pp. 79-91. Bogotá: Siglo del Hombre Editores. http://www,ram-wan.net/restepo/decolonial/14-castro-descolonizar\%20la\%20universidad.pdf

Dussel, E. (2013, octubre). El giro descolonizador. Entrevista videograbada. https://www.youtube. $\mathrm{com} /$ watch? $\mathrm{v}=\mathrm{m} \mid \mathrm{FF} 73 \mathrm{wIMQE}$

Escobar, A. (2005). Más allá del tercer mundo. Universidad de Cauca, Bogotá.

Historiografía (2017). En América Latina: desplazamiento del dominio colonial europeo al dominio colonial y neocolonial de los Estados Unidos. https://hormigacity.blogspot. com/2017/10/paises-que-fueron-colonias-espanolas.html

Maldonado-Torres, N. (2007). Sobre la colonialidad del ser, contribuciones al desarrollo de un concepto, en: Castro-Gómez S. y Grosfoguel, R. (Eds.). El giro decolonial. Reflexiones para una diversidad epistémica más allá del capitalismo global. pp.127-167. Bogotá, Colombia: Siglo del Hombre Editores.

Mignolo, W. (2003). Historias locales / diseños globales. Akal.

Morin, E. (2018). ¿Qué es transdisciplinariedad? http://edgarmorinmultiversidad.org/index.php/ que-es-transdisciplinariedad.html

Nicolescu, B. (1996). La Transdisciplinariedad. Manifiesto. Ediciones Du Rocher. http://edgarmorinmultiversidad.org/index.php/que-es-transdisciplinariedad.html
Quijano, A. (2000a). "Colonialidad del poder y clasificación social", Journal of World-System Research, 11 (2), Riverside.

Quijano, A. (2000b). "Colonialidad del poder, eurocentrismo y América Latina”, en E. Lander (Comp.) La colonialidad del saber. CLACSO.

Quijano, A. (2001). "Colonialidad del poder, globalización y democracia”, En AAW Tendencias básicas de nuestra época. Instituto Pedro Gual.

Quijano, A. (1991, 1992). "Colonialidad y modernidad/racionalidad”. En Perú Indígena. 13 (29).

Quijano, A. y Wallerstein, I. (1992). La americanidad como concepto, o América en el moderno sistema mundial. Revista Internacional de Ciencias Sociales. XLIV, 4, p. 549-557.

Quintero, P. (2010). Notas sobre la Teoría de la colonialidad del poder y la estructuración de la Sociedad en América Latina. Papeles de Trabajo. No. 19-Junio 2010- ISSN 1852-4508. Centro de Estudios Interdisciplinarios y Etnolingüística y Antropología Socio-Cultural. https://core.acuk/ download/pdf/62638027-pdf

Rincón, O. Millán, K. y Rincón, O. (2015),. El asunto decolonial: Conceptos y debates, Año 3, N. ${ }^{\circ}$, pp. 75-95.

Rojas Tudela, F. (2017). Colonialidad, laRazón (Edición Impresa). http://www.la-razon.com/opinion/columnistas/Colonialidad 0 2688331182.html

Significados.com. "Colonia”. https://www.significados.com/colonia/

Vélez Cardona, W. (2014). Hacia una docencia transdisciplinaria en unos estudios Generales Latinoamericanos y Caribeños. Ponencia presentada en el Tercer Seminario Internacional sobre Estudios Generales auspiciada por INTEC y RIDEG, Santo Domingo, República Dominicana, 24 y 25 de julio de 2014. 